Magdalena Mironesko, A. (2020): Esperanto vs. lengua vernácula en China a principios del siglo XX: la problemática y las propuestas reformistas.

Cultura, Lenguaje y Representación, Vol. XXIII, 109-120

ISSN $1697-7750 \cdot$ e-ISSN 2340-4981

DOI: http://dx.doi.org/10.6035/clr.2020.23.7

\title{
Esperanto vs. lengua vernácula en China a principios del siglo XX: la problemática y las propuestas reformistas
}

Esperanto vs. vernacular language in China at the beginning of the 20th century: the problematic and the reform proposals

ALEXANDRA MAGDALENA MironeSKO

UNIVERSIDAD DE GRANADA

Artículo recibido el / Article received: 2019-06-13

Artículo aceptado el / Article accepted: 2020-02-11

RESUMEN: Las diferentes lenguas del mundo han supuesto siempre una barrera y obstáculo a la hora de establecer relaciones internacionales. Es por ello que no han faltado intentos en unificar las lenguas, modificarlas o incluso de crear algunas nuevas para facilitar la comunicación entre distintas naciones. Siendo quizás la más importante el esperanto, esta lengua artificial encontró muchos seguidores, incluso en China, tales como Cai Yuanpei, reformista de gran importancia en los inicios del siglo XX. Sin embargo, dentro de China había otro dilema con respecto a la lengua en el cual se apostaba por la vernacularización de la misma, creando así dos posturas en las que Cai Yuanpei tomaría parte. El objetivo del presente artículo es analizar el contexto histórico y cultural, así como la problemática lingüística observada en la China de principios del siglo XX y las propuestas de los intelectuales más relevantes. Para este fin, se ha utilizado la metodología descriptivo-comparativa, que pone de manifiesto las diferentes visiones de los reformistas sobre la lengua, llegando a la conclusión de que, a pesar de que el esperanto no se impuso como la herramienta comunicativa común, su atracción e influencia convirtieron a China en un epicentro del esperantismo mundial durante las primeras décadas del siglo XX.

Palabras clave: esperanto; lengua china; Cai Yuanpei; reforma; baihua.

ABSTRACT: The different languages of the world have always been a barrier and an obstacle when it comes to establish international relations. That's why there have been attempts to unify the languages, modify them or even create new ones to make the communication easier between different nations. Esperanto maybe being the most important one, this artificial language found lots of followers, even in China, like Cai Yuanpei, a reformist of great importance in the beginning of the 20th century. However, in China there was another dilemma surrounding the vernacularization of the Chinese language, resulting in two campaigns in which Cai Yuanpei would take part. The objective of this article is to analyze the historical and cultural context, as well as the linguistic problems observed in China at the beginning of the 20th century and the proposals of the most relevant intellectuals. For this purpose, the descriptive-comparative methodology that 
highlights the different views of the reformists on the language has been used, reaching the conclusion that, despite the fact that Esperanto did not prevail as the common communication tool, its attraction and influence made China an epicenter of global Esperantism during the first decades of the 20th century.

Key words: Esperanto; Chinese language; Cai Yuanpei; reform; baihua.

Según se escribe -en hebreo y en el capítulo XI del Génesis, primer libro del Pentateuco- los soberbios mesopotámicos súbditos del rey Nimrod, empeñados en la construcción de una torre cerca de la ciudad de Babilonia, que alcanzase los cielos, fueron castigados por el Señor [Yahweh], confundiéndolos en sus comunicaciones orales y diversificando las lenguas, pues (versículo 1): «toda la tierra hablaba la misma lengua y las mismas palabras»; (versículo 6): «[...] son un solo pueblo y todos ellos tienen la misma lengua [...]»; se enfadó y dijo el Señor (versículo 7): «vamos, bajemos y allí confundamos su lengua, para que nadie entienda el lenguaje del otro».

Para corregir tal diversidad y variedad lingüística - causa de tantas guerras, odios, conflictos, problemas, incomprensiones y sinsabores a toda la Humanidad durante siglos y milenios-, el 26 de julio de 1887, el médico oftalmólogo de origen judío Dr. Ludwik Lejzer Zamenhof publicó en Varsovia, en ruso y bajo el pseudónimo de Doktoro Esperanto (=Doctor Esperanzado), un extenso folleto titulado Международныии языкъ. Предисловіе и полный учебникъ. Lingvo internacia. Antaŭparolo kaj plena lernolibro (=Lengua Internacional. Prefacio y libro de estudio completo), después conocido bajo el título Unua Libro o Libro primero, donde describe y expone los principios y fundamentos de una nueva y artificiosa 'lengua internacional' por él creada. Este manual o libro de estudio contenía ya ejemplos de conocidos textos traducidos, como la oración cristiana del Padre nuestro, algunos versículos de la Biblia (del capítulo I de Génesis), una epístola, algunos poemas, así como las dieciséis normas básicas de su estructura gramatical y casi un millar de raíces léxicas. Siguieron otras ediciones en traducciones al inglés (1888, 1889), ídish, hebreo, lituano, sueco (1889), checo, búlgaro, danés, español e italiano (1890) y otras al francés, polaco y alemán. Con el tiempo, la lingvo internacia pasó a denominarse directamente como esperanto, tomándose el pseudónimo del creador como nombre propio de dicha neolengua.

Zamenhof nació el 15 de diciembre de 1859 en Białystok, actualmente Polonia, pero entonces bajo dominio del Imperio Ruso y falleció el 14 de abril de 1917 en Varsovia. Tenía como idioma materno y familiar el ídish o judeoalemán, sociolecto vernáculo de la numerosa población hebrea asquenazí de la Europa Central e Imperio Ruso, pero también hablaba con fluidez el alemán y dominaba, como idiomas nativos o de cultura escolar y académica, el polaco y el ruso; más tarde estudió latín, griego, hebreo, francés e inglés, e incluso español, italiano y otros idiomas. Así que la invención de la nueva lengua que, según él, tenía que unir los pueblos del mundo y solucionar el problema de la confusión lingüística suscitada durante la construcción de la antes mencionada Torre de Babel se basaba en un profundo y amplio conocimiento de diferentes lenguas, pertenecientes a las distintas familias lingüísticas indoeuropeas, tanto clásicas (latín y griego) como modernas (germánicas, eslavas, románicas) y a la semítica hebrea.

Con todo, acaso convenga recordar que esta lingvo internacia de Zamenhof debió competir con su predecesora volapük, creada por el clérigo alemán Johan Martin Schleyer y dada a conocer ocho años antes (mayo de 1879 en la revista Sionsharfe: Monatsblätter für katholische Poesie) bajo el ambicioso lema de una lengua para una humanidad. Se 
impuso finalmente la nueva propuesta de Zamenhof debido a las muchas complicaciones del volapük y la relativa sencillez del esperanto. Su léxico se basa mayormente en los de las lenguas neolatinas y anglogermánicas, la morfosintaxis se aproxima a las lenguas eslavas, los morfemas son invariables, de libre combinación por el usuario y permitiendo gran riqueza en la formación y creación de nuevas palabras, de fonética articulatoria sencilla y regular con acentuación siempre paroxítona y ortografía regular que adjudica a cada sonido un grafema (algunos llevan diacríticos), entre otras características. Esta neolengua funciona como una lengua de tipo aislante, como el chino, mientras que su estructura interna general apunta más bien hacia una tipología aglutinante, como el turco o el japonés.

Los esfuerzos e intereses de Zamenhof por difundir su lingvo internacia cristalizaron en la convocatoria y celebración en Boulogne-sur-Mer (7-12 de agosto de 1905) del primer Universala Kongreso de Esperanto, que reunió a 668 participantes de diversos países y diferentes lenguas. Sin embargo, Zamenhof no pudo acudir debido a la guerra entre Japón y Rusia (1904-1905).

En 1907 -cuando ya el esperanto había triunfado definitivamente sobre el volapük- surgió en París un entusiasta movimiento revisionista, encabezado por el matemático y lingüista Louis Couturat y el esperantista Louis de Beaufront -asimismo conocido como marqués Louis Chevreux-, que proponía una radical reforma de la lingvo internacia de Zamenhof bajo una nueva lengua auxiliar internacional o esperanto mejorado de nombre ido.

Los congresos universales esperantistas se celebran anualmente desde esa fecha, exceptuando las ausencias de algunos años durante las dos guerras mundiales. En España en 1905 los esperantistas organizaron el $5^{\circ}$ Universala Kongreso de Esperanto en Barcelona, con la participación de más de 1500 congresistas. En un emotivo y solemne acto, el rey Alfonso XIII nombró Comendador de la Orden de Isabel la Católica al Dr. Zamenhof. En 1968, en el 53 ${ }^{\circ}$ Kongreso en Madrid asistieron 1769 esperantistas y en 1993 se organizó en Valencia el $78^{\circ}$ con 1863 asistentes. En el pueblo valenciano de Cheste (8200 habitantes) se enseña y habla esperanto desde 1908 y el círculo actualmente cuenta con unos 200 esperantistas.

Sin embargo, el esperanto no fue una lengua del interés exclusivo de los entusiastas occidentales, en especial de los europeos. Al otro lado del continente euroasiático las reformas y el cambio se daban en China, que estaba saliendo de una etapa dinástica milenaria, al tiempo que los reformistas proponían nuevas ideas de modernización y progreso para realizar el tan deseado proyecto de instaurar una moderna república. Un problema ya común de la nueva China que debía de entrar en el siglo XX y estar en contacto con el resto del mundo al mismo nivel era, indudablemente, el de la lengua, la cual había sido prácticamente exclusiva de una selecta minoría que podía permitirse instrucción y educación. La lengua china se caracterizaba en esos momentos por ser un idioma clásico, complejo y con un dificilísimo sistema de escritura, prácticamente inaccesible para el pueblo llano, que había convertido a la mayoría de la población en una masa de analfabetos. Así, a finales del siglo XIX el porcentaje de hombres que sabían leer y escribir no superaba $4 \%$ y entre las mujeres solo una de cada mil no era analfabeta (Коростовец, 1892: 176).

Acaso convenga tener presente cuál era, en aquel momento, la situación de la lengua china para entender el porqué de la necesidad de unas profundas reformas, como por ejemplo, el hecho de que el sistema de escritura de los hanzi (汉字) resultaba ser un método complejo, debido a la gran cantidad de caracteres, trazos, radicales y significados a comprender, memorizar y estudiar. Mientras que todos estos elementos pertenecían a una lengua más clásica y anticuada, había que considerar, por un lado, la necesidad de 
añadir nuevas palabras, así como los más variados préstamos léxicos de origen extranjero $\mathrm{y}$, por el otro, crear los oportunos neologismos para designar las novedades que se iban introduciendo en China en el ámbito de tecnología, ciencia, ingeniería, economía, política, etc.

La sociedad de China, en su camino hacia la modernización, pasando por esa nueva etapa en su historia, requería de un lenguaje contemporáneo y actualizado, incluyendo una nueva y amplia terminología propia de los más diversos campos. No era posible explicar y enseñar los nuevos conocimientos usando únicamente una lengua clásica y anacrónica, incapaz de expresar los conceptos nuevos venidos con los avances de tecnología, ciencia, economía, etc. del nuevo siglo, elementos que durante mucho tiempo quedaron alejados o resultaron de poco interés para China. La carencia de este vocabulario, de usarse tan solo la lengua clásica, supondría una enseñanza incompleta y comprensión deficiente de esos conocimientos, por lo que resultaba de extrema urgencia la adaptación de la lengua china a un estilo propio y que pudiera añadir los necesarios neologismos acordes a la nueva época, razón por la que no pocos reformistas, en busca de una lengua unificada, no solamente para China sino para contactar con el resto del mundo, se interesaron por el esperanto.

En el París de 1907, un grupo de exiliados y estudiantes chinos (Benton, 2007: 92-114) editaron un periódico de ideología anarquista y en lengua esperanto con el título La Novaj Tempoj (新世纪 Xin Shiji = El Nuevo Siglo). En agosto de ese mismo año se celebró en Cambridge el $3^{\circ}$ Universala Kongreso de Esperanto, y en noviembre, el anarquista Li Shizeng (李石曾) proponía en un artículo una radical revolución en los ámbitos de las lenguas y escrituras de China, en el sentido de que había que abandonar los sistemas y lenguas tradicionales y sustituirlos por el alfabeto latino y la neolengua internacional, el esperanto (Kaske, 2008: 381). Tal propuesta provocó inmediatamente una viva polémica en el seno de otros grupos de estudiantes e intelectuales chinos asentados en Europa, encabezados por el círculo parisino de Wu Zhihui (吴稚晖) ${ }^{1}$, discutiendo ellos las formas de introducir el esperanto en China, al considerar que el idioma nacional clásico podría ser un obstáculo en el camino del progreso. Así, Wu Zhihui, proponía en otro escrito reemplazar la cultura tradicional literaria china por otra en esperanto.

Los patriotas de hoy ven la creación de una nación de ciudadanos (guomin 国民) como su misión más importante. Por ello insisten en enseñar en las escuelas primarias solamente la literatura nacional (guowen 国文) y prohibir la enseñanza de las lenguas extranjeras. Esto es lo que ellos aprenden de la experiencia de otros países. Pero dejadnos apartar la espuma de la olla y ver qué clase de literatura nacional es realmente vuestra noble literatura nacional. Simplemente porque este tipo de escrituras no es adecuado para el desarrollo de los principios científicos de la civilización, nos hemos hundido en la barbarie más profunda. Sólo recientemente la gente ha empezado a hablar de construir escuelas modernas. Pero si ahora solamente vertemos las aguas residuales de un cubo a otro, tal educación no es diferente a la de enseñar a los [...] rebeldes Boxers.

(Kaske, 2008: 381)

Sin embargo, en el tan apasionado debate sobre el esperanto la revista El Nuevo Siglo dejó prácticamente de lado todo lo relacionado con la lengua china vernácula. Por

\footnotetext{
${ }^{1}$ Wu Zhihui (1865-1953), lingüista y filósofo, de ideología anarquista a principios del siglo XX, cuando estudiaba en París. Junto a Li Shizeng (李石曾1881-1973), Zhang Renjie (張人傑 1877-1950) y Cai Yuanpei (蔡元培 1868-1940), más tarde conocidos como los Cuatro Ancianos, constituyeron durante los años 20 el núcleo duro de los anticomunistas del Partido Nacionalista.
} 
otra parte, el sabio Zhang Binglin² (章炳麟), en 1908 atacó los puntos de vista de los anarquistas reflejados dicha publicación y repudiaba el uso del esperanto en China, argumentando, desde puntos de vistas lingüísticos y filológicos, que dicha lengua artificial había sido creada en base a las estructuras y léxicos de algunas lenguas europeas. Además, acusaba a los anarquistas chinos de someterse a los dictados e influencias ideológicas de los europeos. A su vez, proponía la creación de una neolengua panasiática (亚洲新语 Yazhou Xinyu) que compartiese elementos básicos de las cuatro grandes lenguas de las culturas y civilizaciones de Asia: chino, sánscrito, persa y árabe, con el objetivo de facilitar la comunicación entre los pueblos orientales (Kaske, 2008: 383).

En las páginas de la revista esperantista La Novaj Tempoj ( $\mathrm{n}^{\circ} 30,18$ enero 1908) aparecieron los artículos de Li Shizeng y Chu Minyi donde ponían de manifiesto que los caracteres chinos y su lento y costoso aprendizaje, elitista por definición, constituían un serio obstáculo para la comunicación y conllevaban atraso e incultura. Una escritura de corte fonético exigiría la eliminación de los dialectos vernáculos y mejor sería sustituir el chino escrito por un idioma internacional como el esperanto (Benton, 2011: 95), aduciendo algunas razones lingüísticas y de orden práctico: a) en muchas lenguas, la escritura no se adapta a la pronunciación, lo que no se da en esperanto, donde a cada grafema o letra (del alfabeto latino con algunas adiciones diacríticas) corresponde un único sonido; b) en esperanto, el acento fónico es siempre paroxítono (es decir, siempre recae en la penúltima sílaba); c) cada palabra posee un único significado, con lo que se evitan las homonimias, el lenguaje figurado, las metáforas y otras confusiones semánticas o de interpretación dudosa, y d) la búsqueda de las palabras en los diccionarios es sumamente fácil, siguiendo el orden alfabético (Benton, 2011: 95).

La recomendación del empleo y difusión del esperanto en China no debía considerarse como una falta de patriotismo, ya que para aspirar a una radical modernización y puesta al día de la gran nación china, en vez de optar por la enseñanza y difusión del inglés u otra lengua culta occidental se debía acceder a las culturas, ciencias y tecnologías extranjeras - mayormente del mundo anglo-germánico de Europa y América- a través del esperanto, lengua perfecta y superior a las demás lenguas naturales, de modo que se recomendaba abandonar el bárbaro y complicado sistema chino de escritura (Benton, 2011: 95).

Quizás el más destacado defensor de este nuevo idioma en el gigante asiático fuera el lingüista, pedagogo y reformista educador chino Cai Yuanpei (1868-1940). Buen conocedor de la complicada situación lingüística de China, Cai Yuanpei fue uno de los más importantes intelectuales involucrados en las reformas de la sociedad china, especialmente en las educativas, y se dio cuenta de las oportunidades que ofrecía el esperanto. Nacido en 1868 en Shaoxing, Cai Yuanpei provenía de una familia con dificultades económicas heredadas tras la muerte de su padre. Sin embargo, siendo todavía muy joven, Cai Yuanpei demostró un gran talento y habilidad para el estudio y el aprendizaje, siendo instruido por los amigos de su padre (Boorman \& Howard, 1970: 295). Bajo la tutela de su tío y de algunos conocidos de su familia, se formó en los Clásicos y aprendió la correcta manera de redactar los ensayos de ocho partes que se solicitaban en los exámenes civiles, de modo que a los veintidós años logró aprobarlos: «Cai había obtenido el mayor honor acorde al escolar de la China tradicional» (Duiker, 1977: 5), demostrando que aún muy joven, se vio impulsado por el conocimiento y aprendizaje, lo que le llevaría, con los años, a reflexionar sobre el estado actual de la educación china, así como de la filosofía confuciana, base del modelo educativo chino,

\footnotetext{
${ }^{2}$ Zhang Binglin (1868-1936), filólogo y crítico literario. Compuso el El origen de la escritura (文始 Wen Shi), el primer diccionario etimológico de la lengua china.
} 
tomando en estas cuestiones al Occidente y su cultura como punto de referencia y paradigma a seguir.

En especial, uno de los principales asuntos relacionados con la educación fue, para Cai Yuanpei, la reforma de la lengua. Él era consciente de que, en todo lo concerniente a ésta, no era el único interesado en proponer radicales, necesarios y decisivos cambios. Mucho antes de su nombramiento como Ministro de Educación de la recién instaurada República de China (1912) y antes de aceptar el cargo de rector de la Universidad de Pekín (1916) había ideado y diseñado toda una serie de bases y elementos para la reforma de la lengua, si bien la vernácula no cobraba gran protagonismo en sus propuestas: él mismo había sido formado en el marco teórico y práctico del sistema clásico confuciano y con un estilo escrito de corte muy tradicional, por lo que, al igual que otros reformistas instruidos en ese campo, le resultaba difícil conceder a la lengua hablada, vernácula, cierto valor utilitario como elemento decisivo para el cambio.

Cuando en 1904 Cai Yuanpei publicó su conocida novela Un sueño de Año Nuevo (新年梦 Xinnian Meng) (Guangyi Li, 2013: 89-104; Andolfatto, 2015: 11-31), redactado en chino vernáculo baihua (白话文 Baihuawen), y seriado en los últimos números de la revista Noticias sobre Rusia (俄事警闻 Eshi Jingwen)(Kaske, 2008: 206), imaginaba en su relato la historia de un ficticio, educado y políglota ciudadano chino que, a lo largo de sus viajes por Europa, habría entrado en contacto con la ideología de los populistasanarquistas narodniki rusos $^{3}$ y llegado a la convicción de que el cosmopolitismo y la unión de todos los pueblos del mundo requerirían toda suerte de sacrificios y esfuerzos, de modo que cada nación -y particularmente la nación china-, en vistas a la tan deseada unificación, debía situarse en posición de igualdad con respecto a todos los demás pueblos y naciones.

Claro está que ese nuevo, educado y cosmopolita ciudadano chino no es otro sino el álter ego del propio Cai Yuanpei, angustiado por el incierto futuro de la lengua vernácula baihua en el marco de un previsible y deseado proceso de modernización de China, concebido, según el propio Cai, en tres fases: en un primer estadio, y una vez conseguida la implantación y consolidación de un nuevo régimen político republicano, se observaría una patente fragmentación y diversidad de lenguas y dialectos entre las clases educadas y la pervivencia de la escritura de la lengua clásica. Las élites dirigentes continuarían usando sus dialectos locales; mientras, la lengua vernácula se expresaría solamente por escrito en las novelas, y oralmente en los discursos públicos y el teatro de contenido político con la finalidad y objetivo de formar y educar ideológicamente a las clases populares en la toma y adquisición de una conciencia nacional y preparar a las masas en vistas de nuevas y progresistas reformas sociales. Una segunda etapa en la modernización y progresos de la nueva China sería la instauración de un tribunal internacional de justicia y una fuerza internacional de pacificación. La lengua de China habría de jugar, pues, un papel esencial en las relaciones internacionales, pero ¿a qué lengua se refiere Cai Yuanpei? En la tercera y última fase, para una era que inicia una comunicación muy amplia, ilimitada, Cai Yuanpei propone un radical cambio de usos lingüísticos mediante la unificación y estandarización de la lengua hablada, del idioma vernáculo baihua (Yu-Fen, 2012: 157-166).

Otro grave problema que preocupaba a Cai Yuanpei era la dispersión y diversidad en el uso de la lengua, pues aparte del chino escrito de los Han, había otras cuatro lenguas oficiales utilizadas por las principales etnias de China del siglo XIX, a saber: tibetano, mongol, árabe y manchú. Esto se traducía en una educación de la lengua escrita

\footnotetext{
${ }^{3}$ Fueron revolucionarios de orientación populista (en ruso 'narod' - народ = 'pueblo') activos entre 18601870.
} 
descentralizada, en la que en algunas escuelas se enseñaba el mongol, pero no la lengua Han o viceversa, lo que presentaba un enorme obstáculo para la comunicación escrita.

Además, otra seria complicación residía en que la lengua china, al contrario de lo que había sucedido con otros idiomas al adaptarse, no presentaba un estilo o tendencia hacia lo vernáculo, es decir, se mostraban patentes y grandes diferencias estilísticas entre la lengua china hablada y la escrita, de carácter más clásico y formal. La gran dificultad del estudio de la escritura china, haciéndola tan inaccesible para muchos, resultaba en que la comprensión de un texto clásico, al equivocarse en la pronunciación de un carácter, fuera errónea, incompleta o un fracaso:

[...] las partes fonéticas y escritas de la lengua china no estaban relacionadas y no había normas claras establecidas para guiar en el entendimiento de la pronunciación de las palabras. La fonética se estudiaba no para la pronunciación sino para la mejor comprensión de los antiguos clásicos con el objeto de esclarecer el significado de las palabras en esos textos. [...] muchos caracteres escritos eran utilizados para indicar múltiples significados, pero con idéntica pronunciación.

(Lin, 2005: 109)

Ya anteriormente se había intentado recopilar y refundir varios sistemas fonéticos, aunque la difusión de los mismos fue más bien escasa. Incluso con la llegada de los misioneros extranjeros, cuyas lenguas podrían suponer un modelo a seguir, en lugar de aprovechar el momento y la favorable coyuntura para crear un conjunto o sistemática recopilación fonética que simplificase la lengua, los chinos continuaron fijando su atención en la longitud del verso y rima de su propia lengua, lo que acabó por separar los caminos de chinos y extranjeros en esos primeros contactos. El territorio chino, de gran extensión, también impedía que se unificara correctamente la lengua dejando así un rastro de dialectos que incluso hoy día son claros ejemplos de la variedad de un mismo idioma, como puede ser el caso del dialecto de Shanghai comparándolo con el dialecto de Chongqin: «Históricamente, la dificultad de transporte había llevado a la formación de muchos dialectos regionales en China que no eran mutuamente inteligibles») (Lin, 2005: $110)$.

Como observa Lin Xie (林捔) (1873-1926), un esperantista contemporáneo de Cai Yuanpei:

Sin embargo, en China no distinguimos entre la lengua escrita antigua y la lengua escrita nacional ni tenemos letras ni escritura fonográfica. Nuestra caótica escritura en sí misma no es fácil de comprender. Más aún, las lenguas habladas y escritas son tan diferentes que uno debe aprender a hablar y escribir por separado. No es de extrañar que nuestros estimados hermanos no puedan invertir tanto tiempo en el estudio.

(Kaske, 2008: 208)

Cai Yanpei tomó como ejemplo el periodo renacentista europeo, donde las lenguas vernáculas locales fueron ganando fuerza sobre la lengua clásica latina, idioma que Cai compara con la clásica china, mostrando así el cambio de dos sistemas cultos y tradicionales hacia unos más comunes y patrimoniales de todo el pueblo. Sus primeros experimentos en la transformación del lenguaje comenzarían a partir de su labor de editor de periódicos, donde Cai hacía una cuidadosa selección de las palabras y del lenguaje dependiendo del lector, de manera que los textos más elegantes y complejos fueran dirigidos a un público más ilustrado, y los más sencillos tuvieran como destinatario a la población de más humilde y básica instrucción (Kaske, 2008: 206).

En esta época y más adelante, mantendría ciertos reparos contra la lengua vernácula. La lengua hablada no se diferenciaba únicamente por los distintos dialectos sino que también era muy distinta dependiendo de la clase social en la que se utilizara: la 
alta sociedad, educada y letrada, acostumbrada a la lengua clásica que era símbolo de su estatus, recurría a un lenguaje que distaba mucho de quienes pertenecían a las clases más bajas, que sí hacían uso de la lengua vernácula. Ésta se utilizaba en novelas, obras de teatro y propaganda para la clase humilde, recurriendo a un estilo más sencillo y directo. Únicamente la lengua clásica como estatus creaba una barrera para la unificación de la lengua, pues mientras la lengua clásica se refería como un símbolo de la alta sociedad intelectual, la vernácula era considerada de clase baja. Esta brecha hizo pensar a Cai Yuanpei que en China era necesaria una lengua única que aglutinase todo: las obras escritas, que pudieran ser compuestas y leídas por todos; la educación, que pudiera llegar a todo el mundo y para todas las clases sociales, de modo que ya no hubiese semejante separación en la sociedad entre unos y otros:

[En segundo lugar] nuestros ancestros no tenían otros cursos de estudio que la lengua nacional. De lo seis a los veinte años, todo lo que uno escribía y leía era la lengua de los antiguos, así que era sencillo de aprender. Ahora hay mucha ciencia que necesita ser estudiada, y si no reducimos el tiempo necesitado para el estudio de la lengua nacional, ¿cómo lo gestionaremos? Además, en el pasado sólo una pequeña minoría aprendía a leer y si en su ambiente invertían un poco de tiempo ¿qué diferencia había? Hoy queremos que todas las personas sean capaces de leer y escribir, así que ¿cómo podemos pedirles que malgasten tanto tiempo?

(Duiker, 1977: 63)

La lengua que podría servir de herramienta para conseguir este ambicioso objetivo podría ser el esperanto, que cumplía su deseo de lengua artificial dirigida a la comunicación internacional. Esta lengua sí constaba de un alfabeto, muy reducido, por lo que Cai Yuanpei confiaba que sería una lengua mucho más sencilla de aprender y además podría representar un nexo no sólo dentro de todos los aspectos sociolingüísticos de China, sino también fuera del país, haciendo uso de este idioma universal que lograría unir a todos los pueblos en esa idea utópica.

Cai Yuanpei consideraba a la sazón que no era conveniente, ni factible, la introducción violenta y forzada del esperanto en China y creía -como otros intelectuales y lingüistas reformistas, entre los que se contaba el escritor Zhou Shuren (周樹人, más conocido por su pseudónimo Lu Xun鲁迅)- que lo más operativo y razonable era, en un primer momento, la reforma de las lenguas vernáculas chinas: a) simplificación de los caracteres escritos y uso de partículas para marcar las partes del discurso hablado; b) cada palabra en esperanto se correspondería a otra china equivalente; c) la escritura correría de izquierda a derecha y en sentido horizontal, y d) las frases y oraciones chinas se adaptarían a las estructuras de las lenguas occidentales. Solamente tras la radical reforma del vernáculo baihua propuesta por Cai Yuanpei, podría un chino aprender esperanto en tres meses (Benton, 2011: 96). Conviene recordar, no obstante, que Cai Yuanpei en enero de 1912 ocupó el cargo de Ministro de Educación durante el primer gobierno republicano de Yuan Shikai (袁世凯) ${ }^{4}$ y consiguió acuerdos para que el esperanto fuese enseñado como lengua opcional -tanto como lengua auxiliar utilitaria en las relaciones internacionales como instrumento de ayuda en el aprendizaje de otras lenguas extranjeras occidentales- en escuelas y facultades universitarias (Benton, 2011: 103).

La lenta implantación del movimiento esperantista en China a partir de 1911 va siguiendo la penetración y evolución de las ideas anarquistas y socialistas de conspicuos intelectuales como Jiang Kanghu (江元虎), Shifu (師傅), Huang Zunsheng (黄尊生), Xu Lunbo (许论博), Ou Shengbai (区声白) y Liang Bingxian (梁冰弦) entre otros (Benton,

\footnotetext{
${ }^{4}$ Renunció a los pocos meses y viajó a Alemania y Francia. Regresó a China en 1916 y en 1917 fue nombrado Rector de la Universidad de Pekín.
} 
2011: 100-103). El 15 de septiembre de 1915 Chen Duxiu (陈独秀) funda y edita en Shanghai la revista Nueva Juventud (新靑年 La Jeunesse), que derivará hacia posturas marxistas-comunistas a partir de 1917. Chen, en aquellos azarosos momentos a finales de 1916 y en medio de una agria polémica entre partidarios y detractores del vernáculo chino y la lengua artificial de Zamenhof, reflejada en las cartas de los lectores en la revista Nueva Juventud vol. 2, n 3, p. 2., igual que antes Cai Yuanpei, consideraba que el estudio del esperanto no era una cuestión urgente. Con todo, los editores y principales colaboradores de la mencionada revista ejercían como docentes en la Universidad de Pekín, de la que Cai Yuanpei era Rector. Éste propuso al veterano esperantista (y anarquista) Sun Guozhang (孙国璋) como profesor de esperanto en la Universidad; en diciembre de 1917 acudían a sus clases cincuenta y tres estudiantes (Dirlik, 1991:173).

Sin embargo, no siempre Cai Yuanpei coincidía con otros intelectuales respecto a este asunto, hallando más bien diferentes opiniones sobre cómo afrontar la problemática lingüística en China. La reforma de la lengua ganaba peso sobre la opción de la instauración de una nueva, en este caso el esperanto. Cai Yuanpei se vería en la posición de inclinarse más a favor de tal idea reformista, iniciando esta nueva etapa apoyando la propuesta de otro intelectual, Liang Qichao (梁启超) (1873-1929) con su estilo de nueva prosa, la cual se usaría, principalmente y como primer paso de este cambio, en la propaganda política (Kaske, 2005: 203). Otro destacado pionero de este movimiento de la nueva lengua fue Chen Tianhua (陈天华) (1875-1905), uno de los primeros en componer una serie de ensayos y tratados revolucionarios con estilo literario vernáculo, usando estos mismos textos como futuras bases para la reforma: «El resultado fue un estilo retórico que era perfectamente adecuado para ser proclamado público» (Kaske, 2005: 166).

Destacaba también el intelectual Hu Shi (胡适) (1891-1962), quien en la revista Nueva Juventud, publicaba sus escritos en los que animaba al uso de la lengua vernácula. Era necesario que la lengua fuera más allá de las clases sociales para convertirse en un elemento de todos. Así, para Hu Shi: «[...] la nueva cultura debe de ser una cultura de masas, y eso significa el uso de la lengua vernácula como medio de expresión en la literatura» (Duiker, 1977: 62).

Siendo Cai Yuanpei entusiasta partidario del esperanto, entra la duda de por qué permitiría, pues, el auge de una lengua vernácula como era el baihua. Aparte de esperantista, Cai Yuanpei destacó como importante educador, especialmente al aceptar el cargo de Rector de la Universidad de Pekín y lograr poco a poco su auge como institución educativa nacional de renombre. En su búsqueda de este objetivo, Cai Yuanpei tomó una postura educativa más democrática, es decir, permitiendo la diversidad de opiniones en la Universidad para convertirla en un centro de debates y de investigación. En su Universidad, por ello, era tan común ver profesores de educación tradicional confuciana que enseñaban los Clásicos como profesores que habían adquirido una formación más moderna y occidental, de tal manera que había diversas opciones y vías de educación, formación y enseñanza. Uno de los profesores que Cai Yuanpei contrató para la Universidad fue, de hecho, Hu Shi, en su puesto de profesor de literatura, quien sí abogaba fuertemente por una reforma de la lengua en lugar de la adaptación de una nueva. El acercamiento de Cai Yuanpei a la problemática de la lengua estaba, por ello, muy unida a la del carácter de aceptación de diversas ideas que aplicaba en la Universidad de Pekín, y al ir ganando fuerza el baihua dentro de la propia Universidad, Cai no podía arremeter contra tal propuesta, respetando sus ideales educativos y aceptándola como medida para la lengua, si bien siempre fue y sería un acérrimo esperantista:

Primero, era la lengua contemporánea, y él sentía que las nuevas ideas debían ser expresadas mediante formas contemporáneas. Si la lengua literaria -la lengua de los 
antiguos- era utilizada para expresar ideas modernas, entonces se perdería muchísimo tiempo en la traducción, y no para un buen propósito.

(Duiker, 1977: 63)

La agria controversia suscitada entre los radicales esperantistas (de ideologías anarquistas-nihilistas) y los reformadores del chino vernáculo se recrudeció a lo largo de los siguientes años. Así, por ejemplo, al esperantista (y por otra parte filólogo y lingüista reformador del chino clásico) Qian Xuantong (钱玄同), se enfrentó el sociólogo Tao Menghe (陶孟和), que consideraba el esperanto (a fin de cuentas una lengua artificiosa, sin componentes asiáticos) como un instrumento de alienación (Benton, 2011: 104) al tiempo que insistía en la íntima relación entre lengua y carácter nacional. A esta polémica se sumaron otros, como el antes mencionado Chen Duxiu (nombrado Decano de la Universidad de Pekín por Cai Yuanpei en 1917), Wu Zhihui y Huang Lingshuang (que comparó el esperanto con el volapük y el idioma neutral, derivado éste del volapük y dado a conocer por Woldemar Rosenberg en 1903), hasta perder virulencia en las páginas de la revista Nueva Juventud en febrero de 1919. El entusiasmo por la lengua artificial internacional de Zamenhof se vio frenado y relegado a un segundo plano de la lucha ideológica en China debido, sobre todo, por la gran decepción que sintieron los revolucionarios izquierdistas-internacionalistas chinos de ideologías anarquistas, marxistas-comunistas, republicanos, etc. tras conocerse los humillantes términos del Tratado de Versalles, firmado en la ciudad francesa tras la Primera Guerra Mundial el 28 de junio de 1919 y que entró en vigor el 10 de enero de 1920. El artículo 156 del Tratado entregaba a Japón la península china de Shandong, lo que dio lugar a violentas manifestaciones y al nacimiento del Movimiento del 4 de Mayo (五四运动). La delegación china, sintiéndose menospreciada, fue la única que no firmó el tratado.

Con todo, a principios de los años 20, Sun Guozhang seguía impartiendo cursos de esperanto en la Universidad de Pekín, con la participación y asistencia de no pocos estudiantes. Y el interés por la lengua de Zamenhof aumentó con la incorporación del celebrado poeta esperantista ucraniano Vasil Yakovich Eroshenko (en ucraniano Василь Якович Срошенко) a dicha Universidad -invitado en febrero de 1922 por Cai Yuanpeidonde dictaba sus conferencias y clases en inglés y no ocultaba sus ideas anarquistas a la vez que abiertamente repudiaba y criticaba el bolchevismo soviético. También fundó su propia escuela de esperanto, contando con la ayuda de Cai Yuanpei y otros prominentes y veteranos miembros del grupo El Nuevo Siglo. En julio-agosto de 1922 participó en el $14^{\circ}$ Universala Kongreso de Esperanto celebrado en Helsinki en calidad de representante de la Cina Esperanto-Ligo. Y a finales de ese año -el 15 de diciembre- y antes de abandonar China, Eroshenko todavía participó en Pekín en un congreso de la citada Cina Esperanto-Ligo para conmemorar el aniversario de Zamenhof. El propio Cai Yuanpei sugirió entonces que el esperanto, en tanto que lengua internacional y neutral, permitiría presentar a China en occidente bajo nuevas y mejores luces y pidió al diplomático chino Wellington Koo (顾维钧) que pronunciase su discurso en esperanto.

Otro ferviente defensor y promotor del esperanto como lengua auxiliar fue el comunista $\mathrm{Hu}$ Yuzhi (胡愈之), quien en la prestigiosa Revista del Este (东方杂志全 Dongfang Zazhi) disertaba sobre la posición internacional de la lengua creada por Zamenhof. También Ou Shengbai y Huang Zunsheng -veteranos esperantistas de ideario anarquista-, participaron en abril de 1922 en los debates y discusiones en la Liga de las Naciones (Ginebra) sobre la adopción del esperanto en las escuelas. Huang residió en Francia hasta 1926 y participó en varios congresos esperantistas europeos representando a China. En el encuentro de Venecia de 1923, como delegado de la Cámara de Comercio 
de Pekín y Tianjin, propuso el esperanto como útil lengua común en los negocios, intercambios y transacciones. En julio-agosto de 1924 participó junto a Cai Yuanpei en el $16^{\circ}$ Universala Kongreso de Esperanto celebrado en Viena, y de nuevo al año siguiente, en el $17^{\circ}$ Kongreso celebrado en Ginebra. En 1924 entró a formar parte del Lingva Komitato y asistió a diversos congresos de ámbito nacional en España, Bulgaria, Rumanía y Yugoslavia (Benton, 2011: 107-109).

Tras las purgas anticomunistas de Chiang Kai-shek (蒋介石) en 1927, Cai Yuanpei y otros veteranos esperantistas apoyaron al líder nacionalista. La revista del Guomindang (中国国民党) de inspiración anarquista Semanal Revolucionario (革命周 报Geming Zhoubao) focalizó inicialmente sus artículos en una viva polémica anticomunista y situó más tarde, en el $\mathrm{n}^{\mathrm{o}}$ 4, de la citada revista, 31 de julio de 1927 (Benton, 2011: 111-113), la cuestión esperantista como la tercera revolución tras el anarquismo (o revolución política) y el comunismo (o revolución económica); el esperantismo sería, pues, la revolución espiritual basada en catorce objetivos dirigidos a construir una sociedad anarco-comunista, una ciencia y cultura basadas en la filantropía, una educación cimentada en el espíritu y principios filantrópicos, la liberación de la Humanidad, la paz permanente, una moralidad basada en la filantropía antes que en la ley, la libre asociación de los pueblos, la libertad individual, una vida estéticamente plena, el amor libre, repudio del nacionalismo y el militarismo, contra la necesidad de la lucha por la existencia, contra cualquier forma de dictadura y contra las dictaduras de clase (Benton, 2011: 110-111).

En la actualidad, la lengua vernácula es la que finalmente se ha instalado en la comunicación contemporánea china. Junto a la posterior simplificación de la escritura, en el último siglo el chino pasó por una serie de procesos mediante los que consiguió mantener su esencia a la par que poder ser una lengua moderna y viva. Aunque el estilo escrito continúe con algunas características de tipo clásico, el propio baihua es la lengua vigente en el día a día, mientras que el esperanto parece ser una lengua de nobles objetivos, pero pospuesta para otro momento. Sin embargo, al igual que Cai Yuanpei en su tiempo y especialmente ante la lengua inglesa, la popularidad del esperanto en China sigue a un ritmo que no se compara al de ningún otro país. Desde el siglo XX, el número de interesados por el esperanto ha crecido enormemente. En los años 80, más de 400,000 chinos formaban parte de la Asociación Esperantista, y aunque la cifra decayó con la introducción y auge de otras lenguas (inglés, español, etc.) la comunidad que estudia y se dedica al esperanto sigue siendo impactante, especialmente si se compara con el número de esperantistas en otros países. Indudablemente, «China is considered by many to be the hope for Esperanto's future» (He, 2017). De este modo, se podría afirmar que si Cai Yuanpei pudiera saberlo, estaría orgulloso por el vigoroso estado del esperanto en China, herencia que sin duda y en alto grado se le puede atribuir a él.

\section{REFERENCIAS}

Andolfatto, Lorenzo. 2015. The chinese utopian novel at the beginning of the twentieth century, 1904-1910. (Tesis doctoral). Venezia: Università Ca' Foscari Venezia y Lyon: Université Lyon III Jean Moulin.

Benton, Gregor. 2011. Chinese Migrants and Internationalism. Forgotten histories, 1917-1945. London-New York: Routledge.

Dirlik, Arif. 1991. Anarchism in the Chinese Revolution. Berkeley \& Los Angeles: The Regents of the University of California. 
Duiker, William. 1977. Ts'ai Yuan-p'ei: Educator of Modern China. United States: The Pennsylvania State University.

Elman, Benjamin y Alexander Woodside. 1994. Education and Society in Late Imperial China, 1600-1900. Berkeley: University of California Press.

Guanyi, Li. 2013. New Year's Dream: A Chinese Anarcho-cosmopolitan Utopia. Utopian Studies, 24 (1): 89-104.

Hao, Ping. 2008. Peking University and the origins of higher education in China. United States: Bridge21 Publications, LLC.

Hayhoe, Ruth. 1992. Education and Modernization: The Chinese Experience. New York: Pergamon Press.

He, Keyao. 2017. Esperanto language grows in China, helps people from different cultures and backgrounds express themselves: http://www.globaltimes.cn/content/1057976.shtml

Hsü, Immanuel C.Y. 2000. The Rise of Modern China. New York: Oxford University Press.

Kaske, Elizabeth. 2008. The Politics of Language in Chinese Education, 1895-1919. Leiden-Boston: Brill.

Коростовец, Иван. 1892. Образование в Китае. Вестник Eвропы, 9: 173-188.

Lin, Xiaoqing Diana. 2005. Peking University: Chinese Scholarship and Intellectuals, 1898-1937. Albany: State University of New York Press.

Schirokauer, Conrad y Amanada Brown. 2006. Breve historia de la civilización china. Barcelona: Edicions Bellaterra.

Yu-Fen, Tai. 戴毓芬. 2012. «La traducción literal del escritor Lu Xun (1881-1936) como impulso a la lengua vernácula (baihua) y a la nueva literatura en China». Estudios de Traducción, 2: 157-166. 